Centro de Referência e

Treinamento em DST/Aids, Coordenação do Programa Estadual DST/Aids-SP, Coordenadoria de Controle de Doenças, Secretaria de Estado da Saúde de São Paulo

Correspondência | Correspondence: Secretaria de Estado da Saúde de São Paulo Av. Dr. Arnaldo, 351, $1^{\circ}$ andar, sala 135 01246-901 São Paulo, SP, Brasil E-mail: bepa@saude.sp.gov.br

Texto de difusão técnico-científica da Secretaria de Estado da Saúde de São Paulo

\section{Recomendações para o funcionamento dos Centros de Testagem e Aconselhamento (CTA) do estado de São Paulo}

\section{Guidelines for Voluntary Counseling and Testing Centers (VCT) in the State of São Paulo, Brazil}

Os Centros de Testagem e Aconselhamento (CTA) são serviços de saúde que, articulados aos demais serviços do Sistema Único de Saúde (SUS), representam uma estratégia importante na promoção da eqüidade de acesso ao aconselhamento e ao diagnóstico do HIV, das hepatites B e C e da sífilis. Atuam também na prevenção dessas e das demais infecções sexualmente transmissíveis (IST), favorecendo segmentos populacionais em situação de maior vulnerabilidade, com respeito aos direitos humanos, à voluntariedade e à integralidade da atenção, sem restrições territoriais.

O CTA Henfil, primeiro do estado de São Paulo, foi criado em 1989 no município de São Paulo. Essa rede vem ampliando-se e, atualmente, é constituída por 97 serviços sediados em 60 municípios prioritários para o controle das IST e da Aids.

No sentido de contribuir para o aprimoramento do trabalho realizado pelos CTA, a coordenação do Programa Estadual de DST/Aids de São Paulo recomenda que esses serviços pautem seu trabalho nos princípios, objetivos e atividades essenciais, população prioritária e parâmetros estruturais, de acesso e de gestão, abaixo descritos.

\section{PRINCÍPIOS}

- Acessibilidade, universalidade e equidade no acesso às ações prestadas pelos CTA, aos insumos de prevenção e aos exames sorológicos.

- Anonimato flexível, confidencialidade e voluntariedade em todos os serviços oferecidos à população.

- Agilidade e resolutividade nas ações prestadas.

- Abordagem interdisciplinar, na perspectiva da atenção integral aos usuários dos serviços.

- Referências inter e intra-setoriais na perspectiva da atenção integral e do acesso universal.
- Articulação com a comunidade em processos de definição e implantação de estratégias para a redução dos contextos locais de vulnerabilidade.

- Respeito e promoção dos direitos humanos e da diversidade sociocultural, principalmente no que se refere à diversidade étnica, de raça e cor, de orientação sexual, ao uso de drogas, à orientação religiosa e às questões de gênero.

- Acolhimento de todos os usuários que chegam ao serviço, levando em consideração a privacidade, a ética, o respeito às diferenças e necessidades trazidas para o CTA.

\section{OBJETIVOS}

- Ampliar o acesso da população em geral, principalmente das populações mais vulneráveis, ao aconselhamento, às ações de prevenção e ao diagnóstico da infecção pelo HIV, sífilis e hepatites B e C.

- Contribuir para a redução das vulnerabilidades ao HIV, às hepatites B e C, à sífilis e outras IST.

- Promover o acesso da população em geral, especialmente dos segmentos populacionais mais vulneráveis, às informações e insumos de prevenção das IST, HIV e hepatites B e C: preservativos masculinos de $52 \mathrm{~mm}$ e $49 \mathrm{~mm}$, gel lubrificante, preservativo feminino e kit de redução de danos.

- Realizar ações de aconselhamento para promover a reflexão sobre as vulnerabilidades, estimular a adoção de medidas de prevenção mais viáveis para cada usuário e reduzir o impacto emocional dos diagnósticos.

- Propiciar a reflexão da comunidade sobre questões relativas à sexualidade e gênero, na perspectiva 
dos direitos sexuais e reprodutivos, e ao uso de álcool e outras drogas, na perspectiva da redução de danos.

- Constituir-se em referência para a demanda por testes sorológicos dos bancos de sangue.

- Estimular o diagnóstico das parcerias sexuais e de outros comunicantes de hepatites B e C, que incluem: indivíduos que compartilham material para uso de drogas; filhos de mãe anti-HCV ou HBs-Ag reagente e indivíduos do mesmo domicílio.

- Apoiar tecnicamente a rede de atenção básica para a implantação e ampliação das ações de aconselhamento, diagnóstico e prevenção das IST, Aids, sífilis e hepatites B e C nos serviços da rede de saúde.

- Encaminhar portadores do HIV, de IST e pessoas com suspeita de hepatites B e/ou C para serviços de saúde, com oferta de acompanhamento até o atendimento na referência. Realizar outros encaminhamentos e orientações que se façam necessários para resolução dos problemas de saúde dos usuários dos CTA.

- Constituir parcerias com instituições locais (organizações não-governamentais, instituições governamentais, universidades e setor privado, entre outros), visando: realização de atividades de prevenção das IST, Aids, sífilis e hepatites B e $\mathrm{C}$, melhoria da qualidade da atenção, ampliação do acesso, formação de profissionais de saúde e desenvolvimento de pesquisa, entre outros.

- Notificar às secretarias municipais e estaduais de saúde os agravos de notificação compulsória. Produzir informações para subsidiar planejamento das ações locais.

\section{POPULAÇÃO PRIORITÁRIA}

- Segmentos populacionais mais vulneráveis, definidas localmente, com participação comunitária, considerando contextos epidemiológicos, socioeconômicos e culturais.

- Gays e outros homens que fazem sexo com homens (HSH), usuários de drogas (UD/UDI), profissionais do sexo, travestis e transexuais são segmentos populacionais reconhecidamente mais vulneráveis, cabendo aos CTA fazer esforços para identificá-las e promover sua inclusão nos serviços.

\section{ATIVIDADES ESSENCIAIS}

- Os CTA deverão oferecer à população os exames necessários para diagnóstico sorológico de HIV, sífilis e hepatites B e C, respeitando os algoritmos estabelecidos pelo Ministério da Saúde, quais sejam:
- Triagem (Elisa) + confirmatório (Western Blot ou imunoblot ou imunofluorescência indireta) para HIV e teste rápido diagnóstico para HIV (TRD HIV)

- VDRL e confirmatório (hemaglutinação - TPHA ou MHA ou outro teste treponêmico: FTA ou Elisa) para sífilis

- $\mathrm{HBsAG}+$ anti-HBc total para hepatite B

- Anti-HCV para hepatite C

- Oferta de teste rápido de diagnóstico anti-HIV (TRD HIV) complementarmente à testagem convencional em todos os CTA do estado de São Paulo.

- Seguindo a Resolução SS-41, de 24 de abril de 2001, considerar como prazo máximo aceitável 15 dias corridos entre a coleta de material e a entrega do resultado aos usuários.

- Aconselhamento individual, coletivo e de casal, inclusive para casais soropositivos, sorodiferentes e do mesmo sexo.

- Atividades internas de prevenção da transmissão do HIV, das hepatites B e C, da sífilis e de outras IST a serem realizadas pelos CTA: aconselhamento; grupos para segmentos populacionais específicos; disponibilização de insumos de prevenção e material informativo; atividades educativas; orientações sobre uso de drogas na perspectiva da redução de danos, inclusive com disponibilização de kits de redução de danos; outras, a serem definidas de acordo com a realidade do serviço.

- Distribuição dos insumos de prevenção de acordo com os seguintes critérios:

- preservativos masculinos de $49 \mathrm{~mm}$ e $52 \mathrm{~mm}$ : para todos os usuários e usuárias do CTA, com acesso facilitado, disponíveis em locais de acesso público, tais como displays e recepção.

- preservativos femininos para todas as mulheres usuárias do CTA, especialmente para aquelas vivendo com HIV/Aids, profissionais do sexo, portadoras de IST, usuárias de drogas e parceiras de usuários de drogas.

- gel lubrificante para profissionais do sexo, travestis, homens que fazem sexo com homens, mulheres com prática sexual anal, pessoas vivendo com HIV/ Aids e mulheres no climatério.

- kits de redução de danos, cuja composição deve ser feita de acordo com a realidade de uso de drogas no contexto em que o CTA está inserido.

- Os preservativos masculinos devem ser de livre acesso nos serviços, estando disponíveis em locais que os usuários possam retirá-los com facilidade, tais como displays e recepção; os demais insumos 
devem ter quantidade negociada com o usuário, respeitando suas necessidades, sua solicitação e sua prática sexual.

- Para facilitar o acesso aos insumos de prevenção, os CTA devem divulgar a sua disponibilidade na unidade por meio de placas e/ou cartazes. Os usuários não deverão ser obrigados a passar por procedimentos prévios, tais como palestras ou consultas, nem deverá ser exigida a identificação daquele que solicitar insumos.

- Atividades extramuros para prevenção da transmissão do HIV, das hepatites B e C, da sífilis e de outras IST a serem realizadas pelos CTA: ações de redução de danos em campo; atividades educativas em instituições; distribuição de material informativo e de insumos de prevenção em locais públicos ou eventos; atividades de prevenção em campo para segmentos mais vulneráveis; aconselhamento; atividades de divulgação dos CTA; CTA volante; outras, a serem definidas de acordo com a realidade do serviço.

- Atividades de CTA volante, definidas como: realização da testagem em campo com aconselhamento e devolução dos resultados em campo; orientações preventivas, disponibilização de insumos de prevenção e de material informativo/educativo.

- Encaminhamento de pessoas com sorologia positiva para o HIV, sífilis e hepatites B e C para os serviços de referência.

- Encaminhamento de população prioritária para vacinação de hepatite B; usuários dos CTA e portadores de alguma DST.

- Resolução dos casos de IST que chegam ao CTA por meio da identificação de queixas ou sinais e sintomas de doença sexualmente transmissível (DST), seguida de encaminhamento e/ou tratamento dos usuários com suspeita ou diagnóstico de DST por meio da abordagem sindrômica.

- Aconselhamento continuado para as pessoas que aguardam os resultados de exames (HIV, sífilis, hepatites) e também para pessoas vivendo com HIV/ Aids, portadores de hepatites e seus familiares, até que sejam encaminhados e atendidos nos serviços de referência para tratamento.

- Capacitação de profissionais da saúde, e outros atores, em temas como aconselhamento e outros definidos conforme o perfil do CTA e dos profissionais do serviço.

- Divulgação das atividades oferecidas pelo CTA para a população geral, nos bancos de sangue, estabelecendo mecanismos formais de encaminhamento dos usuários que ainda procuram esses serviços para se testar.
- Abordagem consentida, entendida como convite para o comparecimento ao serviço de saúde dos usuários que não compareceram para receber os resultados dos testes realizados para HIV e outras IST, independentemente do resultado do exame, assim como para usuários que realizaram o exame sorológico de hepatite $\mathrm{C}$ conforme estabelecido na Instrução Normativa $n^{\circ} 1.626$, do Ministério da Saúde, de 10 de julho de 2007.

- Notificação às secretarias municipais e estaduais da saúde dos seguintes agravos de notificação compulsória: infecção pelo HIV em gestantes e crianças expostas ao risco de transmissão vertical, sífilis em gestante, hepatites B e C.

- Articulação com a rede de saúde (serviços especializados de DST, Aids, saúde mental, hepatites virais e rede básica).

- Articulação com a gestão do Programa Municipal de DST/Aids.

- Articulação com a sociedade civil organizada e com outras instituições.

- Produção de relatórios de informação sobre perfil da demanda atendida, para subsidiar tanto o planejamento das ações do serviço como as diversas esferas de gestão, a partir da utilização do Sistema de Informação de CTA (SI-CTA).

\section{ASPECTOS ESTRUTURAIS DE ACESSO E GESTÃO}

\section{Equipe}

Recomenda-se que os CTA tenham uma equipe mínima de profissionais, com carga horária compatível com a demanda do serviço. A equipe mínima deverá ser composta de:

- coordenador da equipe;

- mínimo de quatro aconselhadores;

- profissionais responsáveis pela coleta;

- recepcionistas;

- apoio administrativo (qualificado para manuseio dos sistemas de informação);

- profissionais de limpeza e

- segurança.

O número de profissionais da equipe deverá ser ampliado de acordo com: atividades desenvolvidas pelo serviço, inserção dos profissionais em outros serviços de saúde no horário de funcionamento do CTA; capacidade instalada; e demanda. 
Outros profissionais também poderão integrar uma equipe complementar, como o agente de prevenção capacitado em redução de danos e o profissional de apoio na área de informática, para geração de informação a partir do SI-CTA.

\section{Perfil do aconselhador}

O aconselhamento poderá ser realizado por profissionais com formação de nível superior ou técnico que estejam devidamente capacitados para a realização dessa atividade. O profissional deverá ter passado por formação específica e adequada para realização da atividade.

Considerando a integração de profissionais de nível técnico na equipe de aconselhadores dos CTA, recomendase que os de nível superior representem pelo menos $50 \%$ dos integrantes da equipe de aconselhadores.

\section{Horário de funcionamento}

Para promoção do acesso da população às suas atividades, é importante que os CTA desenvolvam suas atividades de segunda a sexta-feira, em período integral (manhã e tarde) e horário estendido (após 18 horas ou nos fins de semana), pelo menos uma vez por semana.

\section{Gerência dos serviços}

É importante que os CTA possuam uma coordenação exclusiva para o desenvolvimento de suas atividades (mesmo quando inserido em outros serviços de saúde) e que esteja presente no cotidiano das atividades do serviço, podendo, a depender da estrutura, acumular mais uma função dentro do próprio CTA. 